\title{
Odd-frequency Cooper pairs and zero-energy surface bound states in superfluid ${ }^{3} \mathrm{He}$
}

\author{
S. Higashitani, S. Matsuo, Y. Nagato, and K. Nagai \\ Graduate School of Integrated Arts and Sciences, Hiroshima University, \\ Kagamiyama 1-7-1, Higashi-Hiroshima 739-8521, Japan \\ S. Murakawa, R. Nomura, and Y. Okuda \\ Department of Physics, Tokyo Institute of Technology, Meguro, Tokyo 152-8551, Japan
}

(Dated: May 23, 2018)

\begin{abstract}
We study the odd-frequency Cooper pairs formed near the surface of superfluid ${ }^{3} \mathrm{He}$. The oddfrequency pair amplitude is closely related to the local density of states in the low energy limit. We derive a formula relating explicitly the two quantities. This formula holds for arbitrary boundary condition at the surface. We also present some numerical results on the surface odd-frequency pair amplitude in superfluid ${ }^{3} \mathrm{He}-\mathrm{B}$. Those analytical and numerical results allow one to interpret the midgap surface density of states, observed recently by transverse acoustic impedance measurements on superfluid ${ }^{3} \mathrm{He}-\mathrm{B}$, as the manifestation of the surface odd-frequency state.
\end{abstract}

PACS numbers: 67.30.hp, 67.30.H-, 74.45.+c

\section{INTRODUCTION}

The spin and orbital degrees of freedom of the Cooper pairs are customarily classified into two categories: spinsinglet even-parity and spin-triplet odd-parity. This classification is based on the requirement that the equal-time pair amplitude is antisymmetric under simultaneous exchange of spin and position variables. Berezinskii has proposed a concept of even- and odd-frequency symmetries associated with the relative-time dependence of the pair amplitude. $\frac{1}{\underline{n}}$ The even-frequency state is characterized by the pair amplitude symmetric in frequency, so that it can be subdivided into the same symmetry classes as in the equal-time case. On the other hand, the oddfrequency state can have the symmetries of spin-singlet odd-parity and spin-triplet even-parity, in contrast to the even-frequency state. The even-frequency symmetry class includes almost all the paring states so far discussed. For instance, the spin-singlet $s$-wave state discussed by Bardeen, Cooper, and Schrieffer for superconducting metals,, 2 the spin-singlet $d$-wave state for cuprate superconductors,$\underline{3}$ and the spin-triplet $p$-wave states for superfluid ${ }^{3} \mathrm{He}^{4}$ and for $\mathrm{Sr}_{2} \mathrm{RuO}_{4}{ }^{\frac{5}{\underline{3}}}$ belong to this category. The possibility of the odd-frequency pairing has been proposed in the context of cuprate superconductors ${ }^{6}$ and heavy Fermion superconductors $\underline{?}$ At present, however, the odd-frequency superconductor or superfluid has not yet been established experimentally.

Bergeret, Volkov, and Efetov pointed out that the oddfrequency Cooper pairs are generated by spatial inhomogeneity even without interaction responsible for oddfrequency pairing. $\stackrel{8}{*}$ After that, several theoretical studies have been reported on the odd-frequency states in superconducting junctions $\stackrel{9-11}{-1}$ An intriguing example of the junctions is a diffusive normal metal/spin-triplet superconductor system. In this system, the proximity-induced superconductivity in the diffusive normal metal is dominated by the odd-frequency spin-triplet $s$-wave Cooper pairs because of isotropization by impurity scattering $\underline{\underline{9}}$
Such an anomalous proximity effect is also expected in

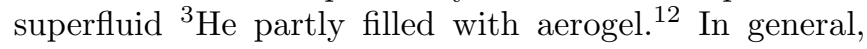
there can coexist even- and odd-frequency Cooper pairs near interfaces or surfaces owing to broken translational symmetry. The inhomogeneous superconducting and superfluid systems therefore provide a good starting point to develop our understanding of the odd-frequency state.

In this paper, we discuss the odd-frequency Cooper pairs generated near the surface of superfluid ${ }^{3} \mathrm{He}$. The spin-triplet $p$-wave state of bulk superfluid ${ }^{3} \mathrm{He}$ is arguably the best understood non-s-wave state $\stackrel{\underline{4}}{\underline{ } \text { It is well }}$ established that the non-s-wave states have pronounced sensitivity to surface scattering $\underline{13-21}$ Unlike the $s$-wave state, the quasiparticle scattering at the surface causes substantial pair-breaking. As a result, the gap function varies in space over several coherence lengths from the surface. In addition, the Andreev bound states are formed at the surface, yielding a characteristic low energy structure in the surface density of states below the bulk gap. The surface Andreev bound states have recently attracted renewed interest from the aspect of their Majorana nature $\underline{21-25}$ The purpose of the present work is to demonstrate the existence of the surface odd-frequency Cooper pairs in superfluid ${ }^{3} \mathrm{He}$. Recently, Aoki et al ${ }^{19}$ and Murakawa et al $\stackrel{20,21}{\underline{2}}$ performed transverse acoustic impedance measurements to probe the surface of superfluid ${ }^{3} \mathrm{He}$ and found an evidence of the low energy density of states due to the formation of the surface Andreev bound states in the B phase of superfluid ${ }^{3} \mathrm{He}$. We show that the observed midgap density of states can also be interpreted as the manifestation of the surface oddfrequency Cooper pairs in superfluid ${ }^{3} \mathrm{He}-\mathrm{B}$.

\section{QUASICLASSICAL THEORY}

Our analysis is based on the quasiclassical Green's function theory of superfluidity and superconductivity $\underline{26}-28$ We apply it to a semi-infinite 
system occupying the space $z>0$. The surface at $z=0$ may have atomic-scale irregularities, though we assume it to be macroscopically flat. The surface roughness of atomic scale, which gives rise to diffuse quasiparticle scattering, is inevitable in actual systems.

The quasiclassical Green's functions, $g$ and $f$, are a function of $(\hat{p}, \epsilon, z)$, where $\hat{p}$ is a unit vector in the direction of the Fermi momentum and $\epsilon$ is a complex energy variable. One can express $g$ and $f$ in the form ${ }^{28}$

$$
g=i \frac{1+\mathcal{F} \tilde{\mathcal{F}}}{1-\mathcal{F} \tilde{\mathcal{F}}}, \quad f=\frac{2 i}{1-\mathcal{F} \tilde{\mathcal{F}}} \mathcal{F},
$$

where $\mathcal{F}(\hat{p}, \epsilon, z)$ and $\tilde{\mathcal{F}}(\hat{p}, \epsilon, z)$ are $2 \times 2$ spin-space matrices and the notation $\tilde{X}$ denotes the transformation $\tilde{X}(\hat{p}, \epsilon, z)=X\left(-\hat{p},-\epsilon^{*}, z\right)^{*}$. The spatial dependence of $\mathcal{F}(\hat{p}, \epsilon, z)$ and $\tilde{\mathcal{F}}(\hat{p}, \epsilon, z)$ are governed by

$$
\begin{aligned}
& i v_{F} \hat{p}_{z} \partial_{z} \mathcal{F}=-2 \epsilon \mathcal{F}+\Delta(\hat{p}, z)+\mathcal{F} \Delta(\hat{p}, z)^{\dagger} \mathcal{F}, \\
& i v_{F} \hat{p}_{z} \partial_{z} \tilde{\mathcal{F}}=2 \epsilon \tilde{\mathcal{F}}-\Delta(\hat{p}, z)^{\dagger}-\tilde{\mathcal{F}} \Delta(\hat{p}, z) \mathcal{F}
\end{aligned}
$$

where $v_{F}$ is the Fermi velocity and $\Delta(\hat{p}, z)$ is a gap matrix. The quasiclassical Green's functions have symmetries

$$
\begin{aligned}
& g(\hat{p}, \epsilon, z)=g\left(\hat{p}, \epsilon^{*}, z\right)^{\dagger} \\
& f(\hat{p}, \epsilon, z)=-f(-\hat{p},-\epsilon, z)^{T} .
\end{aligned}
$$

Here the superscript $T$ denotes matrix transpose. Equations (4) and (5) give the general relation between the retarded Green's function $(\epsilon=E+i 0)$ and the advanced one $(\epsilon=E-i 0)$, and also between the Matsubara Green's functions on the positive and negative imaginary axes in the complex $\epsilon$ plane.

From $g$, one can calculate the local density of states,

$$
n(\hat{p}, E, z)=\operatorname{Im}\left[\frac{1}{2} \operatorname{Tr} g(\hat{p}, E+i 0, z)\right] .
$$

The even- and odd-frequency pair amplitudes are defined in terms of $f$ by

$$
\left.\begin{array}{l}
f^{\mathrm{EF}}(\hat{p}, \epsilon, z) \\
f^{\mathrm{OF}}(\hat{p}, \epsilon, z)
\end{array}\right\}=\frac{1}{2}[f(\hat{p}, \epsilon, z) \pm f(\hat{p},-\epsilon, z)] .
$$

In the following analysis, we consider the unitary states in which $\Delta \Delta^{\dagger} \equiv|\Delta|^{2}$ is proportional to the unit matrix. The unitary condition is satisfied for all of the spinsinglet states and for many of spin-triplet states, e.g., those realized in the $\mathrm{A}$ and $\mathrm{B}$ phases of superfluid ${ }^{3} \mathrm{He}^{4}$ and the two-dimensional chiral $p$-wave state discussed for $\mathrm{Sr}_{2} \mathrm{RuO}_{4} \cdot \frac{5}{6}$

In the unitary states, $\mathcal{F}$ and $\tilde{\mathcal{F}}$ for $z \rightarrow \infty$ take the form

$$
\mathcal{F}^{\infty}=\frac{\Delta^{\infty}(\hat{p})}{\epsilon+i \Omega(\hat{p}, \epsilon)}, \quad \tilde{\mathcal{F}}^{\infty}=\frac{\Delta^{\infty}(\hat{p})^{\dagger}}{\epsilon+i \Omega(\hat{p}, \epsilon)} .
$$

Here $\Delta^{\infty}(\hat{p})=\Delta(\hat{p}, z \rightarrow \infty)$ is the bulk gap matrix and $\Omega(\hat{p}, \epsilon)=\left(\left|\Delta^{\infty}(\hat{p})\right|^{2}-\epsilon^{2}\right)^{1 / 2}$ in which $\left|\Delta^{\infty}(\hat{p})\right|$ corresponds to the bulk energy gap. The $\mathcal{F}^{\infty}$ and $\tilde{\mathcal{F}}^{\infty}$ make the right-hand side of Eqs. (2) and (3) in the bulk region vanish and substitution of them into Eq. (1) gives the well-known bulk solution of the quasiclassical Green's functions, $g^{\infty}=\epsilon / \Omega(\hat{p}, \epsilon)$ and $f^{\infty}=\Delta^{\infty}(\hat{p}) / \Omega(\hat{p}, \epsilon)$.

The boundary problem in the semi-infinite system can be solved in the following way. ${ }^{28}$ To obtain the incoming (outgoing) solution $\mathcal{F}_{\hat{p}_{z}<0}\left(\mathcal{F}_{\hat{p}_{z}>0}\right)$, the differential equation (2) is integrated from right (left) to left (right). Equation (3) for $\tilde{\mathcal{F}}$ is integrated in the opposite direction. The information on the surface is therefore included in the initial values of the outgoing solutions $\left(\mathcal{F}_{\hat{p}_{z}>0}\right.$ and $\left.\tilde{\mathcal{F}}_{\hat{p}_{z}<0}\right)$, while the incoming ones $\left(\mathcal{F}_{\hat{p}_{z}<0}\right.$ and $\left.\tilde{\mathcal{F}}_{\hat{p}_{z}>0}\right)$ are independent of the boundary condition at the surface apart from the implicit dependence through $\Delta(\hat{p}, z)$.

\section{RELATION BETWEEN THE PAIR AMPLITUDE AND THE LOCAL DENSITY OF STATES}

We now consider the retarded Green's functions $(\epsilon=$ $E+i 0)$ for the spin-triplet unitary states and show that the odd-frequency pair amplitude is closely related to the local density of states in the low energy limit. The spintriplet states are characterized by the gap matrix of the form

$$
\Delta(\hat{p}, z)=\boldsymbol{d}(\hat{p}, z) \cdot \boldsymbol{\sigma} i \sigma_{2}
$$

where $\boldsymbol{\sigma}=\left(\sigma_{1}, \sigma_{2}, \sigma_{3}\right)$ is the Pauli matrix. The $d$-vector in the unitary states can be expressed as the product of a real vector and a phase factor:

$$
\boldsymbol{d}(\hat{p}, z)=\boldsymbol{d}_{0}(\hat{p}, z) e^{i \varphi_{0}(\hat{p}, z)} .
$$

In the absence of the magnetic field, the matrix $\mathcal{F}$ does not have a spin-singlet component and we may put

$$
\mathcal{F}=\mathcal{F} \cdot \boldsymbol{\sigma} i \sigma_{2}, \quad \tilde{\mathcal{F}}=-i \sigma_{2} \tilde{\mathcal{F}} \cdot \boldsymbol{\sigma}
$$

It is straightforward to show that the incoming solutions of $\mathcal{F}$ and $\tilde{\mathcal{F}}$ for $|E|<\left|\Delta^{\infty}(\hat{p})\right|$ can be parametrized as

$$
\mathcal{F}_{\hat{p}_{z}<0}=-i \hat{a} e^{i \gamma}, \quad \tilde{\mathcal{F}}_{\hat{p}_{z}>0}=i \hat{a} e^{-i \gamma},
$$

where $\hat{a}$ is a unit vector and $\gamma$ is a real function; those are determined from

$$
\begin{aligned}
& v_{F} \hat{p}_{z} \partial_{z} \hat{a}=2 \cos \left(\gamma-\varphi_{0}\right)\left[\boldsymbol{d}_{0}-\left(\boldsymbol{d}_{0} \cdot \hat{a}\right) \hat{a}\right], \\
& v_{F} \hat{p}_{z} \partial_{z} \gamma=2\left[E-\sin \left(\gamma-\varphi_{0}\right)\left(\boldsymbol{d}_{0} \cdot \hat{a}\right)\right],
\end{aligned}
$$

and the initial values in the bulk region,

$$
\begin{aligned}
& \hat{a}^{\infty}=-\operatorname{sgn}\left(\hat{p}_{z}\right) \boldsymbol{d}_{0}^{\infty} /\left|\boldsymbol{d}_{0}^{\infty}\right|, \\
& \gamma^{\infty}=\varphi_{0}^{\infty}-\operatorname{sgn}\left(\hat{p}_{z}\right) \arcsin \left(E /\left|\boldsymbol{d}_{0}^{\infty}\right|\right) .
\end{aligned}
$$

Equations (1) and (11) yield $g=g_{0}+\boldsymbol{g} \cdot \boldsymbol{\sigma}$ and $f=\boldsymbol{f} \cdot \boldsymbol{\sigma} i \sigma_{2}$ with

$$
g_{0}=\frac{i}{N}\left(1-\mathcal{F}^{2} \tilde{\mathcal{F}}^{2}\right), \quad \boldsymbol{g}=-\frac{2}{N} \mathcal{F} \times \tilde{\mathcal{F}}
$$


and

$$
\boldsymbol{f}=\frac{2 i}{N}\left(\mathcal{F}-\mathcal{F}^{2} \tilde{\mathcal{F}}\right)
$$

where $N=1-2 \mathcal{F} \cdot \tilde{\mathcal{F}}+\mathcal{F}^{2} \tilde{\mathcal{F}}^{2}$. From the above expressions for $g_{0}$ and $\boldsymbol{f}$, one can derive the following relations:

$$
g_{0} \mathcal{F}=\frac{1}{2}\left(\boldsymbol{f}-\mathcal{F}^{2} \tilde{\boldsymbol{f}}\right), \quad g_{0} \tilde{\mathcal{F}}=-\frac{1}{2}\left(\tilde{\boldsymbol{f}}-\tilde{\mathcal{F}}^{2} \boldsymbol{f}\right) .
$$

Combining Eqs. (12) and (19), we obtain

$$
\left(g_{0} \hat{a}\right)_{(\hat{p}, E+i 0, z)}=\frac{i}{2}\left(\boldsymbol{f} e^{-i \gamma}+\tilde{\boldsymbol{f}} e^{i \gamma}\right)_{(\hat{p}, E+i 0, z)} .
$$

Taking the imaginary part of this equation, we get

$$
\left.n(\hat{p}, E, z)\right|_{|E|<\left|\Delta^{\infty}(\hat{p})\right|}=\left|\operatorname{Re}\left[\boldsymbol{D}(\hat{p}, E, z) e^{-i \gamma}\right]\right|
$$

with

$$
\begin{aligned}
\boldsymbol{D}(\hat{p}, E, z) & =\frac{1}{2}\left[\boldsymbol{f}(\hat{p}, E+i 0, z)+\tilde{\boldsymbol{f}}(\hat{p}, E+i 0, z)^{*}\right] \\
& =\frac{1}{2}[\boldsymbol{f}(\hat{p}, E+i 0, z)-\boldsymbol{f}(\hat{p}, E-i 0, z)],
\end{aligned}
$$

where the last equality follows from Eq. (5). At $E=0$, the right-hand side of Eq. (21) can be written in terms of the odd-frequency pair amplitude,

$$
n(\hat{p}, 0, z)=\left|\operatorname{Re}\left[\boldsymbol{f}^{\mathrm{OF}}(\hat{p}, i 0, z) e^{-i \gamma}\right]\right| .
$$

It follows that the observation of a finite density of states at zero energy gives a direct evidence of the existence of the odd-frequency Cooper pairs.

Note that the phase $\gamma$ at $E=0$ arises essentially due to the breaking of time-reversal symmetry. This is because the $d$-vector for the time-reversal invariant states can be taken to be real $\left(\varphi_{0}=0\right)$ and then we see from Eqs. (14) and (16) that $\gamma(E=0)=0$ at any position $z$. Thus, for the time-reversal invariant states, Eq. (23) is reduced to

$$
n(\hat{p}, 0, z)=\left|\operatorname{Re} \boldsymbol{f}^{\mathrm{OF}}(\hat{p}, i 0, z)\right| .
$$

Equation (24) can be applied to superfluid ${ }^{3} \mathrm{He}-\mathrm{B}$, as discussed below.

Before proceeding to discussion on superfluid ${ }^{3} \mathrm{He}$, we briefly mention the relations similar to the above in the case of spin-singlet states with $\Delta(\hat{p}, z)=\Delta_{0}(\hat{p}, z) i \sigma_{2}$ [see also Ref. 29 in which an analog of Eq. (20) for a spinsinglet superconductor can be found]. For $|E|<\left|\Delta_{0}^{\infty}(\hat{p})\right|$, we can put $\mathcal{F}_{\hat{p}_{z}<0}=-i s e^{i \gamma} i \sigma_{2}$ and $\tilde{\mathcal{F}}_{\hat{p}_{z}>0}=i s e^{-i \gamma} i \sigma_{2}$ with $s=\operatorname{sgn}\left[\operatorname{Re} \Delta_{0}(\hat{p}, z)\right]$. Then, we can arrive, in a similar way to the spin-triplet case, at

$$
n(\hat{p}, 0, z)=\left|\operatorname{Re}\left[f_{0}^{\mathrm{OF}}(\hat{p}, i 0, z) e^{-i \gamma}\right]\right|,
$$

where $f_{0}^{\mathrm{OF}}$ is the odd-frequency spin-singlet pair amplitude, i.e., $f^{\mathrm{OF}}=f_{0}^{\mathrm{OF}} i \sigma_{2}$. For the time-reversal invariant states, we can again take $\gamma(E=0)$ to be zero.

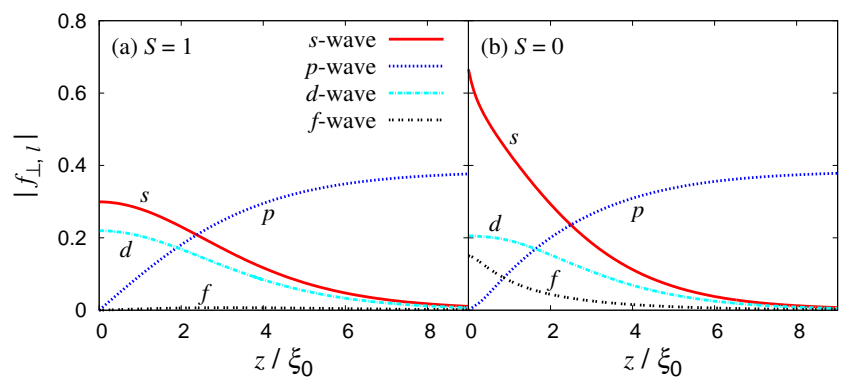

FIG. 1. (Color online) Spatial dependence of $\left|f_{\perp, l}(E+i 0, z)\right|$ at $E=0.5 \Delta_{B}$ in the semi-infinite superfluid ${ }^{3} \mathrm{He}$-B at temperature $T=0.2 T_{c}\left(T_{c}\right.$ : superfluid transition temperature). The surface specularity $S$ is taken to be (a) $S=1$ (specular limit) and (b) $S=0$ (diffusive limit). Self-consistently determined gap functions $\Delta_{\|, \perp}(z)$ are used in the calculations.

Let us turn to the surface odd-frequency state in superfluid ${ }^{3} \mathrm{He}-\mathrm{B}$. The $d$-vector in the semi-infinite superfluid ${ }^{3} \mathrm{He}-\mathrm{B}$ has the form ${ }^{14}-\underline{18}$

$$
\boldsymbol{d}=\left(\Delta_{\|}(z) \hat{p}_{x}, \Delta_{\|}(z) \hat{p}_{y}, \Delta_{\perp}(z) \hat{p}_{z}\right) .
$$

Here $\Delta_{\|, \perp}(z)$ are spatially dependent gap functions, both of which tend for $z \rightarrow \infty$ to the isotropic bulk gap of the B phase, $\left|\Delta^{\infty}(\hat{p})\right| \equiv \Delta_{B}$. Since $\Delta_{\|, \perp}(z)$ can be taken to be real, this state is an example of the time-reversal invariant spin-triplet states.

The $p$-wave gap functions $\Delta_{\|, \perp}(z)$ have the following spatial structure depending on the boundary condition at the surface $\underline{14}-18$ When the surface is specular, the perpendicular component $\Delta_{\perp}(z)$ is strongly suppressed near the surface by the destructive interference effect due to quasiparticle reflection, while the parallel component $\Delta_{\|}(z)$ is slightly enhanced to compensate the condensation energy lost by the suppression of $\Delta_{\perp}(z)$. At rough surface causing diffuse quasiparticle scattering, the parallel component also suffers from the interference effect, so that both of $\Delta_{\|}(z)$ and $\Delta_{\perp}(z)$ are suppressed. The numerical results for $\Delta_{\|, \perp}(z)$ determined self-consistently from the gap equation can be found in Refs. 1418 .

The self-consistent solution of $\boldsymbol{f}$ takes the form

$$
\boldsymbol{f}=\left(f_{\|} \cos \phi, f_{\|} \sin \phi, f_{\perp}\right),
$$

where $\phi=\arctan \left(\hat{p}_{y} / \hat{p}_{x}\right)$ is the azimuthal angle of $\hat{p}$. The pair amplitudes $f_{\|, \perp}$ are a function of $\left(\hat{p}_{z}, \epsilon, z\right)$ and are independent of $\phi$ because of the rotational symmetry around the surface normal.

To illustrate the existence of the surface odd-frequency Cooper pairs in superfluid ${ }^{3} \mathrm{He}-\mathrm{B}$, let us consider the pair amplitude $f_{\perp}\left(\hat{p}_{z}, \epsilon, z\right)$ and its partial-wave components

$$
f_{\perp, l}(\epsilon, z)=\left\langle f_{\perp}\left(\hat{p}_{z}, \epsilon, z\right) P_{l}\left(\hat{p}_{z}\right)\right\rangle_{\hat{p}},
$$

where $P_{l}\left(\hat{p}_{z}\right)$ is the Legendre polynomial and $\langle\cdots\rangle_{\hat{p}}$ denotes the angle average over the Fermi surface. It follows from the general symmetry relation (5) that the even- $l$ (odd- $l$ ) components have the symmetry of odd 


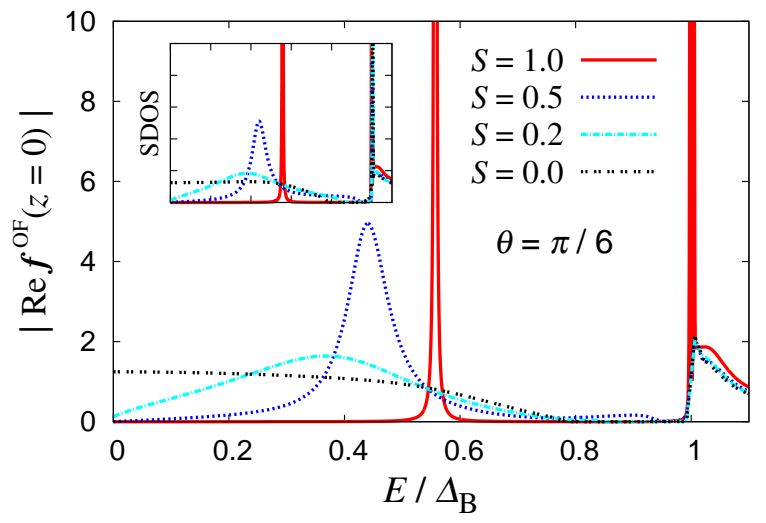

FIG. 2. (Color online) $\left|\operatorname{Re} \boldsymbol{f}^{\mathrm{OF}}(\hat{p}, E+i 0, z=0)\right|$ in the semiinfinite superfluid ${ }^{3} \mathrm{He}-\mathrm{B}$ at $T=0.2 T_{c}$ as a function of $E / \Delta_{B}$. The inset is the surface density of states, $n(\hat{p}, E, z=0)$. The two quantities are independent of the azimuthal angle $\phi=$ $\arctan \left(\hat{p}_{y} / \hat{p}_{x}\right)$ because of the rotational symmetry around the surface normal. The polar angle $\theta=\arccos \left(\hat{p}_{z}\right)$ is taken to be $\pi / 6$. The numerical results are shown for several values of the surface specularity $S$.

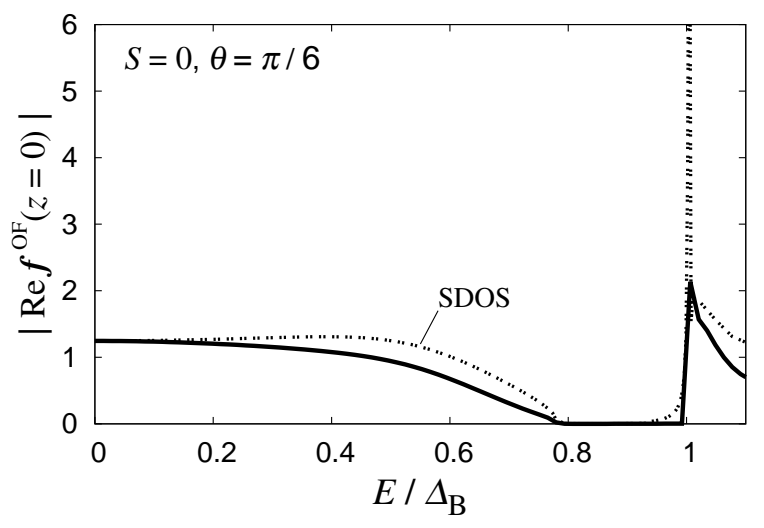

FIG. 3. $\left|\operatorname{Re} \boldsymbol{f}^{\mathrm{OF}}(\hat{p}, E+i 0, z=0)\right|$ shown in Fig. 2 for the diffusive limit $(S=0)$ is compared with the surface density of states, $n(\hat{p}, E, z=0)$.

(even) frequency. In Fig. 1, we plot the magnitude of the self-consistent solution of $f_{\perp, l}(E+i 0, z)$ at $E=$ $0.5 \Delta_{B}$ as a function of $z$ scaled by the coherence length $\xi_{0}=v_{F} / 2 \pi T_{c}$. The rough surface effect is taken into account using the random $S$-matrix theory 17,27 The surface roughness is parametrized by specularity $S(0 \leq$ $S \leq 1 ; S=1$ corresponds to the specular surface and $S \rightarrow 0$ to the diffusive limit where the quasiparticles are scattered isotropically) $\underline{\underline{20,21}}$ The odd-frequency even$l$ Cooper pairs have substantial amplitudes at the surface in both of the specular $(S=1)$ and diffusive $(S=0)$ lim- its. At specular surface, in particular, $f_{\perp}$ has only the odd-frequency components. This is due to the reflection symmetry $f_{\perp}\left(\hat{p}_{z}, \epsilon, 0\right)=f_{\perp}\left(-\hat{p}_{z}, \epsilon, 0\right)$ at the specular surface.

In Figs. 2 and 3 , the $E$ dependence of $\left|\operatorname{Re} f^{\mathrm{OF}}\right|$ for $\epsilon=$ $E+i 0$ at the surface of superfluid ${ }^{3} \mathrm{He}-\mathrm{B}$ is shown together with the surface density of states (SDOS), $n(\hat{p}, E, 0)$. The two quantities have quite similar $E$ dependence. In the limit $E \rightarrow 0$, the both coincide exactly with each other, as we have shown analytically [see Eq. (24)].

In Fig. 2 the sharp peak (solid line) below the bulk gap $\left(E / \Delta_{B}<1\right)$ corresponds to the Andreev bound state formed near the specular surface $(S=1)$. As the specularity $S$ decreases, the peak is broadened and the density of states around zero energy increases. Such low energy excitations in superfluid ${ }^{3} \mathrm{He}-\mathrm{B}$ have recently been observed by transverse acoustic impedance measurements $\underline{20,21}$ In the experiment, the specularity is controlled by coating the surface with thin ${ }^{4} \mathrm{He}$ layers. The specularity dependence of the surface midgap density of states is detected as a distinctive change in a peak structure found in the frequency dependence of the impedance below the bulk gap $\Delta_{B} \stackrel{18,20,21}{=}$ This experiment gives a strong evidence of not only the formation of the surface Andreev bound states but also the existence of the surface odd-frequency Cooper pairs.

\section{CONCLUSION}

Based on the experimental results ${ }^{20.21}$ of the transverse acoustic impedance in superfluid ${ }^{3} \mathrm{He}$ and on the formula (24) relating the odd-frequency pair amplitude to the zero-energy density of states, we have demonstrated that the odd-frequency Cooper pairs are formed near the surface of superfluid ${ }^{3} \mathrm{He}-\mathrm{B}$. The surface odd-frequency state is the origin of the anomalous proximity effect predicted for a diffusive normal metal/spin-triplet superconductor junction 9 and an analogous system composed of aerogel and superfluid ${ }^{3} \mathrm{He}-\mathrm{B}, \frac{12}{}$ Since the pairing symmetry of bulk superfluid ${ }^{3} \mathrm{He}$ is well established, its surface or interface provides an ideal environment for studying the physics of the odd-frequency state.

\section{ACKNOWLEDGMENTS}

We would like to thank Y. Tanaka and Y. Asano for helpful discussions. This work was supported in part by a Grant-in-Aid for Scientific Research (No. 21540365) and the "Topological Quantum Phenomena" Grant-in-Aid for Scientific Research on Innovative Areas (No. 22103003) from MEXT of Japan.
${ }^{1}$ V. L. Berezinskii, JETP Lett. 20, 287 (1974)
2 J. Bardeen, L. N. Cooper, and J. R. Schrieffer, Phys. Rev. 108, 1175 (1957) 
${ }^{3}$ C. C. Tsuei and J. R. Kirtley, Rev. Mod. Phys. 72, 969 (2000)

4 D. Vollhardt and P. Wölfle, The Superfluid Phases of Helium 3 (Taylor \& Francis, London, 1990)

5 A. P. Mackenzie and Y. Maeno, Rev. Mod. Phys. 75, 657 (2003)

6 A. Balatsky and E. Abrahams, Phys. Rev. B 45, 13125 (1992)

7 Y. Fuseya, H. Kohno, and K. Miyake, J. Phys. Soc. Jpn. 72, 2914 (2003)

8 F. S. Bergeret, A. F. Volkov, and K. B. Efetov, Phys. Rev. Lett. 86, 4096 (2001)

9 Y. Tanaka and A. A. Golubov, Phys. Rev. Lett. 98, 037003 (2007)

10 Y. Tanaka, A. A. Golubov, S. Kashiwaya, and M. Ueda, Phys. Rev. Lett. 99, 037005 (2007)

11 Y. Tanaka, Y. Tanuma, and A. A. Golubov, Phys. Rev. B 76, 054522 (2007)

12 S. Higashitani, Y. Nagato, and K. Nagai, J. Low Temp. Phys. 155, 83 (2009)

13 V. Ambegaokar, P. G. deGennes, and D. Rainer, Phys. Rev. A 9, 2676 (1974)

14 L. J. Buchholtz and G. Zwicknagl, Phys. Rev. B 23, 5788 (1981)

15 L. J. Buchholtz, Phys. Rev. B 33, 1579 (1986)

16 W. Zhang, J. Kurkijärvi, and E. V. Thuneberg, Phys. Rev. B 36, 1987 (1987)
17 Y. Nagato, M. Yamamoto, and K. Nagai, J. Low Temp. Phys. 110, 1135 (1998)

18 K. Nagai, Y. Nagato, M. Yamamoto, and S. Higashitani, J. Phys. Soc. Jpn. 77, 111003 (2008)

19 Y. Aoki, Y. Wada, M. Saitoh, R. Nomura, Y. Okuda, Y. Nagato, M. Yamamoto, S. Higashitani, K. Nagai, Phys. Rev. Lett. 95, 075301 (2005)

20 S. Murakawa, Y. Tamura, Y. Wada, M. Wasai, M. Saitoh, Y. Aoki, R. Nomura, Y. Okuda, Y. Nagato, M. Yamamoto, S. Higashitani, K. Nagai, Phys. Rev. Lett. 103, 155301 (2009)

21 S. Murakawa, Y. Wada, Y. Tamura, M. Wasai, M. Saitoh, Y. Aoki, R. Nomura, Y. Okuda, Y. Nagato, M. Yamamoto, S. Higashitani, K. Nagai, J. Phys. Soc. Jpn. 80, 013602 (2011)

22 S. B. Chung and S.-C. Zhang, Phys. Rev. Lett. 103, 235301 (2009)

23 Y. Nagato, S. Higashitani, and K. Nagai, J. Phys. Soc. Jpn. 78, 123603 (2009)

24 G. E. Volovik, JETP Lett. 91, 201 (2010)

25 Y. Tsutsumi, M. Ichioka, and K. Machida, Phys. Rev. B 83, 094510 (2011)

26 J. W. Serene and D. Rainer, Phys. Rep. 101, 221 (1983)

27 Y. Nagato, S. Higashitani, K. Yamada, and K. Nagai, J. Low Temp. Phys. 103, 1 (1996)

28 M. Eschrig, Phys. Rev. B 61, 9061 (2000)

29 Yu. S. Barash, Phys. Rev. B 61, 678 (2000) 